\title{
HOW MANY RATIONAL POINTS DOES A RANDOM CURVE HAVE?
}

\author{
WEI HO
}

\begin{abstract}
A large part of modern arithmetic geometry is dedicated to or motivated by the study of rational points on varieties. For an elliptic curve over $\mathbb{Q}$, the set of rational points forms a finitely generated abelian group. The ranks of these groups, when ranging over all elliptic curves, are conjectured to be evenly distributed between rank 0 and rank 1, with higher ranks being negligible. We will describe these conjectures and discuss some results on bounds for average rank, highlighting recent work of Bhargava and Shankar.
\end{abstract}

\section{INTRODUCTION}

The past decade has seen a resurgence of classical techniques such as invariant theory and Minkowski's geometry of numbers applied to problems in number theory. In particular, the newly coined phrase arithmetic invariant theory loosely refers to studying the orbits of representations of groups over rings or nonalgebraically closed fields, especially the integers $\mathbb{Z}$ or the rational numbers $\mathbb{Q}$. Combining such ideas with improved geometry-of-numbers methods has proved fruitful for some questions in arithmetic statistics (another neologism!), the subject of which concerns the distribution of arithmetic quantities in families.

Perhaps the earliest example of this phenomenon is well known to many number theorists: using Gauss's study of the relationship between equivalence classes of binary quadratic forms and ideal classes for quadratic rings Gau01, Mertens and Siegel determined the asymptotic behavior of ideal class groups for quadratic fields Mer74, Sie44. In other words, Gauss found an arithmetic interpretation for the orbits of the group $\mathrm{GL}_{2}(\mathbb{Z})$ acting on the space $\operatorname{Sym}^{2}\left(\mathbb{Z}^{2}\right)$ of integer binary quadratic forms. Then Mertens's and Siegel's results use the geometry of numbers to "count" those orbits, thereby counting the arithmetic objects parametrized.

Obtaining similar results for binary cubic forms took many more years. Delone and Faddeev found that equivalence classes of integer binary cubic forms are in correspondence with isomorphism classes of cubic rings [DF64, and then Davenport and Heilbronn used the geometry of numbers to compute asymptotics for cubic rings and for cubic fields [DH69]. For example, they proved that the number of isomorphism classes of cubic fields with discriminant between $-X$ and $X$ is

$$
\frac{1}{3 \zeta(3)} X+o(X)
$$

where $\zeta(s)$ is the Riemann zeta function. Since class field theory relates certain cubic fields with 3 -torsion ideal classes of quadratic fields, Davenport and Heilbronn

Received by the editors May 23, 2013.

2010 Mathematics Subject Classification. Primary 11G05, 14H52. Secondary 11G30, 14H25. 
also applied a sieve to obtain the average size of the 3-part of the ideal class group of quadratic fields. In fact, for many years, this was the only proved case of the Cohen-Lenstra heuristics for how ideal class groups are distributed [CL84].

The next major step in arithmetic invariant theory was the program started by Wright and Yukie, who showed that for many representations with a simple invariant-theoretic property, the orbits over a field correspond to field extensions WY92, KY97, Yuk97. Their approach to the resulting statistical questions was to study properties of these representations' zeta functions, as defined by Sato and Shintani [SS74]. While asymptotic results related to some of the representations have been successfully obtained by this method (see, e.g., DW88, KY02, Tan08, TT11]), these zeta functions are quite complicated and difficult to analyze in general Yuk93.

More recently, Bhargava has made vast progress on both sides of this picture, first with a series of papers Bha04a, Bha04b, Bha04c, Bha08 that gave many more examples of parametrizations by integer orbits of representations, including those from Wright and Yukie's work. He also improved the geometry-of-numbers techniques in order to obtain asymptotics for quartic and quintic rings, as well as another case of the (generalized) Cohen-Lenstra heuristics Bha05, Bha10. These results have even led to surprising corollaries, such as the fact that quartic fields whose Galois group is the dihedral group $D_{4}$ make up a positive proportion of all quartic fields when ordered by discriminant 1

The heart of this article describes how this strategy - of combining arithmetic invariant theory and the geometry of numbers to study statistical questions - is successfully applied by Bhargava and Shankar in BS10a to the case of binary quartic forms, leading to results about the distribution of ranks of elliptic curves.

We start, in Section 2 with background material about rational points on curves, especially elliptic curves; this section may be safely skipped by experts, of course. Section 3 discusses precise conjectures related to the distribution of ranks of elliptic curves; for someone not familiar with arithmetic statistics, this section should give a flavor of some types of questions asked - as well as the complications that arise in the subject. The nucleus of the article is Section 4, where we sketch the main ideas behind the theorems of Bhargava and Shankar from BS10a. Finally, Section 5 explains other related developments from the last few years, including interesting corollaries and generalizations, e.g., to higher genus curves.

\section{RATiOnAL POINTS ON VARIETIES}

Finding solutions to polynomial equations is one of the oldest problems in mathematics. Over the last few centuries, mathematicians have formalized the questions and established rigorous language to discuss different variations of this simple idea. While we have made tremendous strides in understanding the structure of these solutions in the last few decades, there remain many fundamental open questions, which lie at the forefront of modern arithmetic geometry.

For the simplest case, let $f\left(x_{1}, \ldots, x_{n}\right)$ be a polynomial with coefficients in $\mathbb{Q}$. We may ask for rational solutions to $f=0$, i.e., numbers $a_{1}, \ldots, a_{n} \in \mathbb{Q}$ such that $f\left(a_{1}, \ldots, a_{n}\right)=0$. To phrase the question more geometrically, let $X$ be the

\footnotetext{
${ }^{1}$ In contrast, when ordering algebraic numbers of degree 4 by the size of the coefficients of the monic minimal polynomials, Hilbert's irreducibility theorem implies that those that generate fields with Galois group $S_{4}$ have density 1.
} 
variety associated to $f$, which may be viewed geometrically as the zero locus of $f$, or solutions to $f=0$, in $\mathbb{C}^{n}$. Then $X$ will be $(n-1)$-dimensional, if $f$ is not a constant. Our problem may be restated as finding rational points on $X$, the set of which is denoted $X(\mathbb{Q})$. More specifically, we may ask questions such as the following:

- Does there exist a single rational point on $X$ ?

- Can we describe all rational points on $X$ ?

- If there are only finitely many rational points, can we enumerate them?

- If $X(\mathbb{Q})$ is an infinite set, what structure does $X(\mathbb{Q})$ have?

If we instead use any finite number of polynomials $f_{1}, \ldots, f_{m} \in \mathbb{Q}\left[x_{1}, \ldots, x_{n}\right]$, we define the analogous variety $X$ to be the common zero locus in $\mathbb{C}^{n}$ of all of the polynomials $f_{i}$. For general choices of $f_{i}$, the dimension of $X$ will be $n-m$, if nonnegative, and 0 otherwise; the rule of thumb is that each polynomial condition imposed should reduce the dimension of $X$ by 1 .

Remark 2.1. While we restrict our attention to varieties defined over $\mathbb{Q}$, i.e., defined by polynomials with coefficients in $\mathbb{Q}$, many of the results that we will discuss have analogues over other number fields.

Even when $X$ is one-dimensional, mathematicians have not yet fully understood how many rational points are on $X$ ! In this article, we focus on this case, where $X$ is a curve.

2.1. Genus and the trichotomy. The arithmetic and the geometry of algebraic curves rely heavily on an invariant called the genus. The genus of a curve may be defined in many ways, but the most intuitive definition is topological. A smooth curve $X$ as defined above may be thought of as a Riemann surface 2 with finitely many punctures; after taking an appropriate compactification by filling these punctures, the resulting compact Riemann surface has a topological genus, which is essentially the number of "holes" or "handles". For example, a complex curve that is homeomorphic to a sphere (after compactification) has genus 0 , while a genus 1 curve looks like the surface of a donut (see Figure 1).

For simplicity, we assume in the sequel that our curves $X$ are compact 3 have no singularities 4 and are connected.
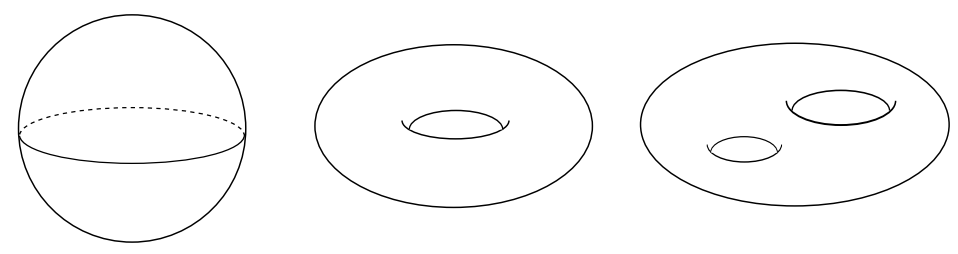

Figure 1. From left to right: curves of genus 0,1 , and 2.

\footnotetext{
${ }^{2}$ The complex points of a smooth curve form a two-dimensional real manifold.

${ }^{3}$ In other words, we always implicitly work with projective curves.

${ }^{4}$ Intuitively, a singularity on a curve is a point where it is not smooth, like a node or a cusp. More precisely, it is a point with more than one tangent direction along the curve.
} 
TABLE 1

\begin{tabular}{c|ccc} 
& genus 0 & genus 1 & genus $\geq 2$ \\
\hline curvature & positive & zero & negative \\
canonical bundle & anti-ample & trivial & ample \\
Kodaira dimension & $\kappa=-\infty$ & $\kappa=0$ & $\kappa=1$ \\
automorphism group & 3-dimensional & 1-dimensional & finite \\
rational points & Hasse principle & finitely generated & finitely many
\end{tabular}

Many properties of curves are heavily influenced by whether a curve has genus 0 , genus 1 , or genus $\geq 2$. Table 1 gives a glimpse of how this trichotomy appears in different areas of mathematics, e.g., ranging from differential geometry to arithmetic.

We will now discuss the last row of this table in more detail.

Curves of genus 0 . For genus 0 curves, there are either no rational points at all or infinitely many. It is fairly easy to determine which case applies to any given curve by the Hasse principle or local-to-global principle: a genus 0 curve $X$ has a rational point if and only if it has a point everywhere locally, which means that the equations defining $X$ have a solution over the real numbers $\mathbb{R}$ and the $p$-adic numbers $\mathbb{Q}_{p}$ for all primes $p$. The nonexistence of a $\mathbb{Q}_{p}$-point is always due to an obstruction modulo a power of the prime $p$.

Example 2.2. Let $X$ be the curve given by the vanishing of the polynomial $f=$ $x^{2}+y^{2}-3$. If there exists a rational solution to $f=0$, by clearing denominators, there are relatively prime integers $r, s$, and $t$ such that $r^{2}+s^{2}=3 t^{2}$. Because squares of integers are congruent to 0 or 1 modulo 4 , reducing the equation modulo 4 shows that $r^{2}$ and $s^{2}$ are both congruent to 0 modulo 4 . This in turn implies that all three integers are even, which is a contradiction. Therefore, the equation $f=0$ has an obstruction modulo 4 , implying that $X$ has no point over the 2 -adic integers $\mathbb{Z}_{2}$ or even over $\mathbb{Q}_{2}$, and thus no rational point.

In fact, checking for local obstructions may be completed in a finite number of steps. Any curve of genus 0 over $\mathbb{Q}$ is isomorphic to a (compactified) plane conic defined by the vanishing of a polynomial of the form

$$
a x^{2}+b y^{2}+c,
$$

where $a, b$, and $c$ are squarefree, pairwise relatively prime integers. A theorem of Legendre implies that (11) has a rational solution if and only if $a, b$, and $c$ do not all have the same sign, and $-a b$ is a square modulo $c,-b c$ is a square modulo $a$, and $-a c$ is a square modulo $b$.

If there is a single rational point $P$ on a conic, then all other rational points come from intersecting the conic with a line of rational slope through $P$.

Example 2.3. If $X$ is given by $x^{2}+y^{2}-2=0$, then by inspection, the point $(x, y)=(1,1)$ lies on $X$. All other points are parametrized by drawing lines of rational (or infinite) slope $r$ through $(1,1)$, and a simple computation shows that $X(\mathbb{Q})$ is the union of the point

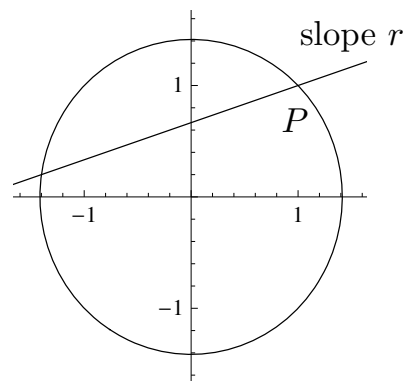


$(1,-1)$ and the points

$$
\left(\frac{r^{2}-2 r-1}{r^{2}+1}, \frac{-r^{2}-2 r+1}{r^{2}+1}\right)
$$

for all $r \in \mathbb{Q}$.

There are also fast algorithms implemented in computer algebra software to find all solutions (see, e.g., CR03).

Curves of genus at least 2. Mordell's 1922 conjecture Mor22 predicted that there could only be finitely many rational points on a curve of genus $\geq 2$; it was proved by Faltings [Fal83] (as a corollary to an even more powerful theorem):

Theorem 2.4 (Faltings 1983). Let $X$ be a curve of genus at least 2 over $\mathbb{Q}$. Then the set $X(\mathbb{Q})$ of rational points is finite.

The original proof uses deep ideas from the theory of $p$-divisible groups, Arakelov theory, and moduli theory, and later proofs and improvements by Vojta Voj91, Faltings [Fal91], Bombieri Bom90, and others use Diophantine approximation methods. None of the proofs, however, are effective in the sense of giving a list of the points in $X(\mathbb{Q})$. In practice, a combination of techniques - including Chabauty's method, Brauer-Manin obstructions, and descent - often are enough to produce the points in $X(\mathbb{Q})$. In $\$ 5.3$, we will outline recent progress on bounding the number of rational points on curves of genus $\geq 2$.

Curves of genus 1 . The case of genus 1 curves is the richest arithmetically, the most complicated, and the most mysterious to this day. A genus 1 curve defined over $\mathbb{Q}$ may have no rational points at all, finitely many, or infinitely many - and it is generally difficult to determine which! Techniques for other genera, such as the Hasse principle, no longer apply, e.g., there are plenty of genus 1 curves which have points everywhere locally but no global rational point.

Genus 1 curves over $\mathbb{Q}$ with a given rational point are known as elliptic curves. Section 2.2 will describe the structure of rational points on elliptic curves in more detail.

2.2. Rational points on elliptic curves. An elliptic curve over $\mathbb{Q}$ is isomorphic to the projective closure of the zero locus of a Weierstrass equation

$$
y^{2}+a_{1} x y+a_{3} y=x^{3}+a_{2} x^{2}+a_{4} x+a_{6}
$$

with all $a_{i} \in \mathbb{Q}$. When defining a nonsingular curve, equation (2) may be transformed (over $\mathbb{Q}$ ) into short Weierstrass form

$$
y^{2}=x^{3}+A x+B
$$

for $A, B \in \mathbb{Q}$ with nonzero discriminant $\Delta=-16\left(4 A^{3}+27 B^{2}\right)$; the nonvanishing of the discriminant ensures that the curve is nonsingular. There is a marked rational point "at infinity", which is denoted $O$. We say that the elliptic curve given by (3) has height

$$
\operatorname{ht}(E):=\max \left(4|A|^{3}, 27 B^{2}\right) .
$$

Many such equations define isomorphic elliptic curves. In particular, for $t \in \mathbb{Q}^{\times}$, scaling $x$ and $y$ by $t^{-2}$ and $t^{-3}$, respectively, in equation (3) gives the new equation

$$
y^{2}=x^{3}+t^{4} A x+t^{6} B .
$$



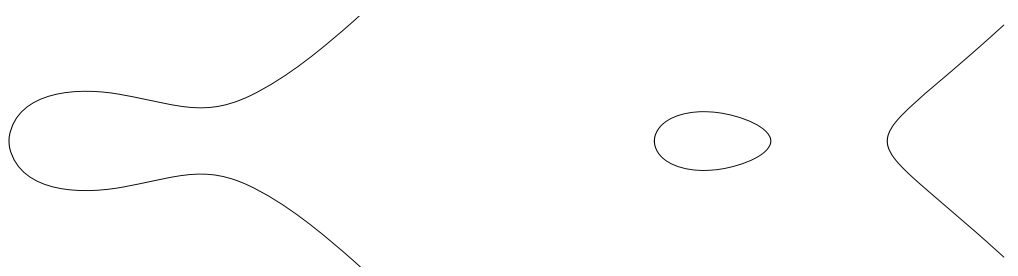

Figure 2. The real points of the elliptic curves $y^{2}=x^{3}-x+1$ (left) and $y^{2}=x^{3}-x$ (right).

In other words, the group $\mathbb{Q}^{\times}$acts on the space of all equations of the form (3) with nonzero discriminant. To choose one representative equation from each $\mathbb{Q}^{\times}$orbit, we define minimal Weierstrass equations to be those of the form (3), with $A, B \in \mathbb{Z}$ and the condition that there is no prime $p$ such that $p^{4}$ divides $A$ and $p^{6}$ divides $B$. Each elliptic curve over $\mathbb{Q}$ has a unique minimal Weierstrass model, and we will call its discriminant the minimal discriminant of the elliptic curve.

For a Weierstrass equation, the solutions lying in a given field have a rich structure. The complex points of an elliptic curve make up a one-holed torus, as discussed earlier. The real points are smooth curves in $\mathbb{R}^{2}$ with one or two components; see Figure 2

Group Law. A beautiful and incredibly useful fact is that the set of solutions in a given field forms a group! An even more powerful statement for rational points is given by a theorem of Mordell Mor22]:

Theorem 2.5 (Mordell 1922). The set $E(\mathbb{Q})$ of rational points of an elliptic curve $E$ defined over $\mathbb{Q}$ forms a finitely generated abelian group, i.e.,

$$
E(\mathbb{Q})=\mathbb{Z}^{r} \oplus E(\mathbb{Q})_{\text {tors }}
$$

for some nonnegative integer $r$ and finite abelian group $E(\mathbb{Q})_{\text {tors }}$.

The group structure on the points of an elliptic curve uses the point $O$ at infinity as the identity element, and it is most easily described geometrically. For the graph of an elliptic curve in short Weierstrass form, as seen in Figure 3, the line $L$ through any two points $P_{1}$ and $P_{2}$ will intersect a third point $P_{3}$ by Bezout's theorem or by direct calculation. The vertical line through $P_{3}$ intersects another point on the elliptic curve, which is the composition $P_{1}+P_{2}$ of $P_{1}$ and $P_{2}$.

In other words, the three (not necessarily distinct) intersection points $P_{1}, P_{2}$, and $P_{3}$ of any line $L$ with the elliptic curve sum to the identity in the group law. The identity point $O$ may be one of these points, e.g., a vertical line intersects $O$, a point $P$, and its negative. Moreover, if $P_{1}$ and $P_{2}$ are rational points, then the line $L$ has rational slope, so $P_{1}+P_{2}$ is also a rational point.

For an elliptic curve $E$ over $\mathbb{Q}$, the torsion subgroup $E(\mathbb{Q})_{\text {tors }}$ of $E(\mathbb{Q})$ is fairly well understood, by the following deep theorem of Mazur [Maz77].

Theorem 2.6 (Mazur 1977). For an elliptic curve $E$ defined over $\mathbb{Q}$, the torsion subgroup $E(\mathbb{Q})_{\text {tors }}$ is one of the following groups:

$$
\begin{array}{cl}
\mathbb{Z} / d \mathbb{Z} & \text { for } 1 \leq d \leq 10 \text { or } d=12, \\
\mathbb{Z} / 2 \mathbb{Z} \times \mathbb{Z} / 2 d \mathbb{Z} & \text { for } 1 \leq d \leq 4 .
\end{array}
$$




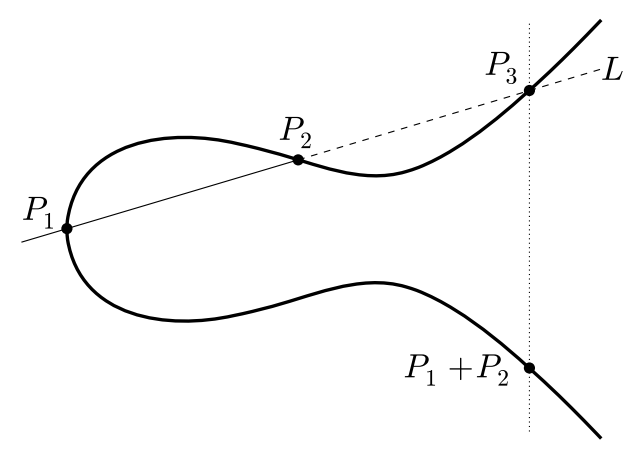

Figure 3. The group law on an elliptic curve.

By Theorem 2.6 and Hilbert's irreducibility theorem, almost al 5 elliptic curves have a trivial torsion subgroup. Moreover, there are explicit methods to quickly compute the torsion subgroup for any given elliptic curve. For example, the rational 2 -torsion points of an elliptic curve in short Weierstrass form are determined by factoring the right-hand side cubic polynomial of (3) over $\mathbb{Q}$.

Example 2.7. The elliptic curve $y^{2}=x(x-1)(x-2)$ has rational 2-torsion subgroup $\mathbb{Z} / 2 \mathbb{Z} \times \mathbb{Z} / 2 \mathbb{Z}$, consisting of the points $(0,0),(1,0),(2,0)$, and $O$ (as the identity). The elliptic curve $y^{2}=x\left(x^{2}+x+1\right)$ has only the rational 2 -torsion points $(0,0)$ and $O$.

In contrast, the rank of $E(\mathbb{Q})$, denoted $r$ in (4), is much more mysterious! It is not even known if the rank can be arbitrarily large; the current record (due to Elkies [Elk07]) is an elliptic curve of rank at least 28. It is quite difficult in general to rigorously prove that a given elliptic curve has a certain rank, though $L$-function computations often give conjectural answers, as we describe very briefly below.

Analytic rank. The $L$-function $L(E, s)$ of an elliptic curve $E$ over $\mathbb{Q}$ is a Dirichlet series given by an Euler product formula involving the number $N_{p}$ of $\mathbb{F}_{p}$-points on the reduction of $E$ over $\mathbb{F}_{p}$, for all primes $p$ :

$$
L(E, s):=\prod_{\operatorname{good} p} \frac{1}{1-a_{p} p^{-s}+p^{1-2 s}} \prod_{\operatorname{bad} p} \frac{1}{1-a_{p} p^{-s}}=\sum_{n \geq 1} a_{n} n^{-s},
$$

where $a_{p}=1+p-N_{p}$ for "good" primes $p$ and $a_{p}=-1,0$, or 1 for a finite set of "bad" primes $p$. By the work of Wiles and others Wil95, TW95, BCDT01, the $L$-function extends to an entire function on the complex plane, and $\Lambda(E, s):=$ $\operatorname{cond}(E)^{s / 2}(2 \pi)^{-s} \Gamma(s) L(E, s)$ satisfies a functional equation

$$
\Lambda(E, s)=u_{E} \Lambda(E, 2-s),
$$

where $\operatorname{cond}(E)$ is an invariant of $E$ called the conductor. The root number $u_{E}$ is either +1 or -1 .

The order of vanishing of this $L$-function $L(E, s)$ at $s=1$ is the analytic rank of $E$. The Birch and Swinnerton-Dyer (BSD) conjecture BSD63, BSD65 claims that the analytic rank of $E$ is equal to the rank of $E$ defined earlier. A more

\footnotetext{
${ }^{5}$ Here, "almost all" means that the density of curves, as defined in 3.1 with trivial torsion subgroup is 1 , when ordered by height.
} 
refined version in fact gives a formula for the coefficient of the leading term in the Taylor expansion at $s=1$ in terms of arithmetic invariants of $E$, including the Tate-Shafarevich group $\amalg(E)$, which will be discussed in 3.3 .

Thus, assuming BSD, one may study the rank of an elliptic curve by understanding its analytic rank, e.g., by computing the apparent order of vanishing of $L(E, s)$ at $s=1$.

\section{RANKS OF ELLIPTIC CURVES}

Most of the remainder of this article is devoted to conjectures and results on the distribution of ranks for elliptic curves. In other words, if we choose a random elliptic curve, what do we expect its rank to be?

3.1. Densities and averages. In order to formulate our question rigorously, we need to specify what is meant by "random" in this setting. We first need an ordering for the (infinite) set of elliptic curves, usually by some sort of invariant; possibilities include ordering elliptic curves up to isomorphism by conductor or by minimal discriminant, or ordering short Weierstrass equations by height or discriminant.

In all these cases, there are only finitely many objects (elliptic curves up to isomorphism or short Weierstrass equations with integral coefficients, for example) with the absolute value of that invariant bounded by any positive number $X$. When the invariant is the discriminant or the conductor, this finiteness is due to Siegel's classical theorem on the finiteness of $S$-integral points on elliptic curves [Sie66]. We may thus define quotients like the following:

$$
\mathrm{P}(\text { invariant }, X, \mathrm{rk}=i):=\frac{\#\{\text { elliptic curves } E \text { with invariant } \leq X \text { and } \operatorname{rank} i\}}{\#\{\text { elliptic curves } E \text { with invariant } \leq X\}},
$$

where the invariant could be the conductor, absolute value of the discriminant, or the height, for example. Then we might ask whether this quantity converges as $X$ tends to infinity, and if so, we may consider the limit

$$
\mathrm{P}(\text { invariant, } \mathrm{rk}=i):=\lim _{X \rightarrow \infty} \mathrm{P}(\text { invariant }, X, \operatorname{rk}(E)=i)
$$

as the density of elliptic curves, ordered by that invariant, with rank $i$ (or equivalently, the probability an elliptic curve has rank $i$ ). We may define a lower density or upper density by instead using liminf or limsup, respectively.

We then define the average rank for elliptic curves, ordered by an invariant, as

$$
\lim _{X \rightarrow \infty} \sum_{i \geq 0} i \cdot \mathrm{P}(\text { invariant }, X, \mathrm{rk}=i)=\lim _{X \rightarrow \infty} \frac{\sum_{\operatorname{invariant}(E) \leq X} \operatorname{rk}(E)}{\sum_{\operatorname{invariant}(E) \leq X} 1}
$$

if this limit exists. Again, a lower average or an upper average is defined using liminf or limsup, respectively; we will sometimes call these the limsup and the liminf of the average rank.

We may also define averages or higher moments for distributions of other quantities associated to elliptic curves in an analogous way. 
3.2. The Minimalist Conjecture. The basic conjecture for the distribution of ranks of elliptic curves is based on the philosophy that elliptic curves should not have any more points than they must.

The widely believed Parity Conjecture, which is a consequence of a weak form of $\mathrm{BSD}$, asserts that the parity of the rank of an elliptic curve is equal to the parity of the analytic rank, which is even exactly when $u_{E}=+1$. It is also strongly expected that the root numbers $u_{E}$ have probability $1 / 2$ of being +1 , and probability $1 / 2$ of being -1 . We are therefore led to the following:

Minimalist Conjecture. The densities of elliptic curves having rank 0 and having rank 1 are both exactly $1 / 2$.

Although no ordering is specified in the statement above, it is conjectured for any reasonable ordering, such as the examples given in 3.1 . Note that the Minimalist Conjecture implies that the density of rank $i$ elliptic curves, for $i \geq 2$, is 0 .

The first version of the conjecture was stated in the 1979 work of Goldfeld Gol79] for quadratic twist families of elliptic curves. Given an elliptic curve $E$ in short Weierstrass form (3), define its quadratic twist $E_{d}$ by a nonzero squarefree integer $d$ as the elliptic curve $y^{2}=x^{3}+d^{2} A x+d^{3} B$. Then Goldfeld's conjecture Gol79] asserts that for a fixed elliptic curve $E$, the average rank of the elliptic curves $E_{d}$ is $1 / 2$, when ordered by $|d|$, that is,

$$
\lim _{X \rightarrow \infty} \frac{\sum_{|d| \leq X} \operatorname{rk}\left(E_{d}\right)}{\sum_{|d| \leq X} 1}=\frac{1}{2},
$$

where the sums are over nonzero squarefree integers $d$. Much work has been done towards this conjecture for quadratic twist families; see Silverberg's survey [Sil07.

The Minimalist Conjecture for all elliptic curves and for quadratic twist families is also supported by the philosophy of Katz and Sarnak [KS99] and later random matrix theory computations and heuristics of Keating and Snaith [KS00], Conrey, Keating, Rubinstein, and Snaith [CKRS02, Watkins Wat08, and others. See [BMSW07,Poo12] for excellent surveys of many aspects of this conjecture.

At various points since Goldfeld's work, the conjecture has been disbelieved, mostly because computations have not seemed to support it. For example, in [KS99, the data of Kramarz and Zagier [ZK87] (extended later by Watkins Wat07]) for a special family of elliptic curves is noted to have a large number of higher rank elliptic curves, with the suggestion that computational capabilities were not yet powerful enough to reflect the true distribution.

The more general computations for the family of all elliptic curves ordered by conductor, by Brumer and McGuinness [BM90, Stein and Watkins [SW02, Cremona Cre06, and Bektemirov, Mazur, Stein, and Watkins [BMSW07, also display a surprisingly large percentage of higher rank elliptic curves. As a result, their data imply asymptotics for average ranks that appear significantly higher than $1 / 2$; see Figure 4, It has been suggested that when restricted to these computational ranges, the data shows the strong effects of secondary terms for the asymptotic number of curves of given rank up to conductor $X$.

Theoretical work on this conjecture is perhaps more optimistic. Brumer Bru92. showed that, assuming the generalized Riemann hypothesis (GRH), the limsup of the average analytic rank of all elliptic curves ordered by height is bounded above by 2.3. Thus, also assuming BSD, Brumer's result implies that the lim sup of the average rank is bounded above by 2.3. This bound was improved by Heath-Brown 


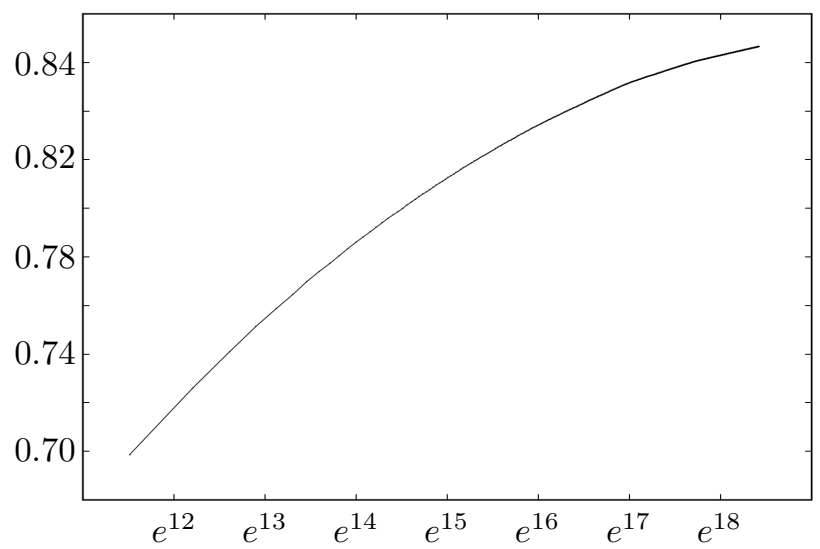

Figure 4. Average rank of elliptic curves in the Stein-Watkins database, up to conductor $e^{18}$. Data and graph by Bektemirov, Mazur, Stein, and Watkins [BMSW07].

HB04 to 2, and then by Young [You06] to 25/14, still assuming BSD and GRH. Young's bound was the first theoretical result implying that a positive proportion of elliptic curves have rank 0 or 1, assuming BSD and GRH.

Finally, the recent work of Bhargava and Shankar BS10a gives an unconditional upper bound:

Theorem 3.1 (Bhargava and Shankar 2010). The limsup of the average rank of elliptic curves over $\mathbb{Q}$, ordered by height, is bounded above by $3 / 2$.

They consider elliptic curves in short Weierstrass form with integral coefficients, and the theorem holds both for all such Weierstrass equations and for only minimal ones. Much of the remainder of this article will focus on this theorem, as well as generalizations and corollaries; see also Poonen's Bourbaki exposé Poo12 for an excellent detailed exposition of [BS10a].

3.3. Selmer groups. Studying the Selmer group of an elliptic curve is one of the only currently known methods to establish an upper bound on its rank. This method is used for both computations for individual curves (e.g., Cremona's mwrank program Cre12) and results for all curves together (as in Theorem 3.1).

The utility of Selmer groups comes from two facts: first, they are finite and often computable; and second, the size of the Selmer group of an elliptic curve gives an upper bound for its rank. More precisely, for a prime $p$, the $p$-Selmer group $\operatorname{Sel}_{p}(E)$ of an elliptic curve $E$ is an elementary abelian $p$-group, i.e., isomorphic to the product of a nonnegative number of $\mathbb{Z} / p \mathbb{Z}$ 's, and its $p$-rank $\operatorname{rk}_{p}\left(\operatorname{Sel}_{p}(E)\right)$ is the number of factors of $\mathbb{Z} / p \mathbb{Z}$. Then

$$
\operatorname{rk}_{p}\left(\operatorname{Sel}_{p}(E)\right) \geq \operatorname{rk}(E) .
$$

Bhargava and Shankar [BS10a] prove Theorem 3.1 by combining (5) for $p=2$ and the following stronger result:

Theorem 3.2 (Bhargava and Shankar 2010). The average number of elements of the 2 -Selmer group for elliptic curves over $\mathbb{Q}$, ordered by height, is 3 . 
Definitions. The content of this subsection may be found in most introductory textbooks on elliptic curves, such as [Sil92, §X.4]. This subsection may be safely skipped at a first reading; it is more important to understand how to access elements of the $p$-Selmer group, as described in the next subsection.

We define the $p$-Selmer group for an elliptic curve $E$ over $\mathbb{Q}$ and a prime $p$. One of the motivating ideas behind the definition is that local computations are often much more feasible than global ones; we also saw this idea in action in 2.1 during the discussion of genus zero curves.

Because the points of $E$ over any field form a group, there is a multiplication-by- $p$ map $E(\overline{\mathbb{Q}}) \stackrel{p}{\longrightarrow} E(\overline{\mathbb{Q}})$, which is surjective and whose kernel is the $p$-torsion subgroup $E(\overline{\mathbb{Q}})[p]$ of $E(\overline{\mathbb{Q}})$. The Galois group $\operatorname{Gal}(\overline{\mathbb{Q}} / \mathbb{Q})$ acts on each of these groups, so the short exact sequence

$$
0 \rightarrow E(\overline{\mathbb{Q}})[p] \rightarrow E(\overline{\mathbb{Q}}) \rightarrow E(\overline{\mathbb{Q}}) \rightarrow 0
$$

of $\operatorname{Gal}(\overline{\mathbb{Q}} / \mathbb{Q})$-modules induces a long exact sequence of Galois cohomology, from which we extract the first row of the commutative diagram (6) below.

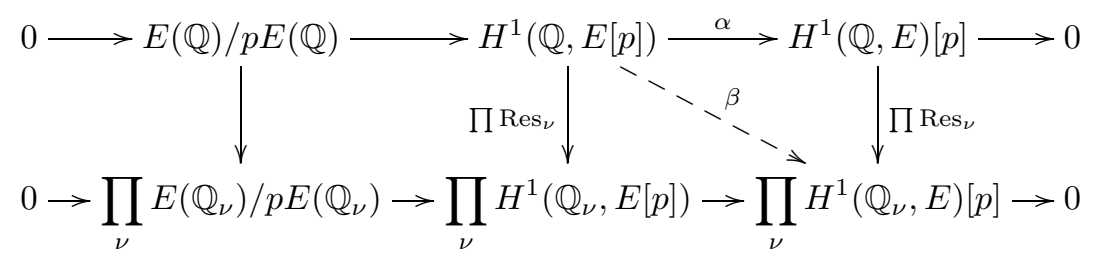

The analogous procedure over each local completion $\mathbb{Q}_{\nu}$ for primes $\nu$ (including $\mathbb{Q}_{\nu}:=\mathbb{R}$ for $\left.\nu=\infty\right)$ gives the second row of (6). The left-most vertical map is given by the inclusions of $E(\mathbb{Q})$ into each $E\left(\mathbb{Q}_{\nu}\right)$, and the latter two vertical maps are products over all primes $\nu$ of the usual restriction maps $\operatorname{Res}_{\nu}: H^{1}(\mathbb{Q}, A) \rightarrow$ $H^{1}\left(\mathbb{Q}_{\nu}, A\right)$ for $A=E[p]$ and $A=E$. Then we make the following definitions:

(i) The $p$-Selmer group $\operatorname{Sel}_{p}(E)$ of $E$ is the kernel of the map $\beta$ in (6) .

(ii) The Tate-Shafarevich group $\amalg(E)$ is the kernel of the map

$$
\prod_{\nu} \operatorname{Res}_{\nu}: H^{1}(\mathbb{Q}, E) \rightarrow \prod_{\nu} H^{1}\left(\mathbb{Q}_{\nu}, E\right) .
$$

Applying the Snake Lemma to a variant of (6) gives the key exact sequence

$$
0 \rightarrow E(\mathbb{Q}) / p E(\mathbb{Q}) \rightarrow \operatorname{Sel}_{p}(E) \rightarrow \amalg(E)[p] \rightarrow 0,
$$

which leads to the inequality (5).

Visualizing elements of Selmer groups. Elements of the Tate-Shafarevich group $\amalg(E)$ and the $p$-Selmer group $\operatorname{Sel}_{p}(E)$ of an elliptic curve $E$ over $\mathbb{Q}$ may be concretely realized using genus one curves and their Jacobians.

The Jacobian $\operatorname{Jac}(C)$ of a genus 1 curve $C$ is the connected component of its automorphism group 6 It is also a curve of genus 1 , and if $C$ has a rational point, then the Jacobian of $C$ is in fact isomorphic to $C$ over $\mathbb{Q}$. As any automorphism group of course has a group structure, including an identity element, the Jacobian of $C$ is an elliptic curve! We call a genus 1 curve $C$ a torsor, or principal homogeneous

\footnotetext{
${ }^{6}$ This definition only works for curves of genus 1 . More generally, the Jacobian is defined to be the dual of the moduli space of degree 0 line bundles.
} 
space, for $\operatorname{Jac}(C)$. In other words, a torsor for an elliptic curve $E$ is a genus 1 curve with an action of $E$ that is simply transitive over $\overline{\mathbb{Q}}$.

The elements of $H^{1}(\mathbb{Q}, E)$ may be thought of as isomorphism classes of torsors for $E$, that is, genus 1 curves $C$ over $\mathbb{Q}$ with an isomorphism of $\operatorname{Jac}(C)$ with $E$. A trivial torsor is isomorphic to $E$ itself, meaning that it has a rational point, and similarly, a torsor $C$ that maps to 0 under $\operatorname{Res}_{\nu}$ has a point in $\mathbb{Q}_{\nu}$.

Therefore, the elements of the Tate-Shafarevich group $\amalg(E)$ are exactly those torsors, up to isomorphism, that have points over every local completion $\mathbb{Q}_{\nu}$, also known as locally soluble. The nonzero elements of $\amalg(E)$ are locally soluble torsors without a global rational point, implying that they fail the Hasse principle.

Elements of the $p$-Selmer group may be represented as locally soluble torsors $C$ for $E$, along with a degree $p$ line bundle on $C$ (or equivalently, a rational degree $p$ divisol 7 on $C$ ). This degree $p$ line bundle on $C$ is equivalent to remembering an algebraic map from $C$ to $(p-1)$-dimensional projective space. As we will describe in more detail in later sections, this description of $p$-Selmer elements may be made yet more explicit for small values of $p$.

Table 2 summarizes these interpretations of the groups for an elliptic curve $E$ over $\mathbb{Q}$.

Heuristics and other work. In the last several years, several heuristics have been developed for the distributions for the Tate-Shafarevich group and $p$-Selmer groups for elliptic curves over $\mathbb{Q}$ (and over other number fields).

Delaunay's heuristics [Del01, Del07] for the distribution of Tate-Shafarevich groups generalizes the ideas behind the Cohen-Lenstra-Martinet heuristics for class groups of number fields CL84,CM87. The main idea is that Tate-Shafarevich groups appear as random finite abelian groups with nondegenerate alternating bilinear pairings, weighted by the inverse of the size of the automorphism group.

The work of Poonen and Rains [PR12] models $p$-Selmer groups as intersections of random maximal isotropic subspaces in an infinite-dimensional quadratic space over $\mathbb{F}_{p}$. They obtain conjectural distributions for $p$-Selmer groups of elliptic curves (and abelian varieties). All of the currently known theoretical results on average sizes of Selmer groups, such as Theorem 3.2, agree with their heuristics. Moreover, the predictions of Poonen and Rains are consistent with the combination of the Minimalist Conjecture and Delaunay's heuristics (using (77) to relate Selmer groups, ranks, and Tate-Shafarevich groups).

\section{TABLE 2}

\begin{tabular}{|c|c|}
\hline Group & Elements (up to isomorphism) \\
\hline$H^{1}(\mathbb{Q}, E)$ & torsors $C$ for $E$ \\
\hline$\amalg(E)$ & locally soluble torsors $C$ for $E$ \\
\hline $\operatorname{Sel}_{p}(E)$ & $\begin{aligned} \text { pairs }(C, L): & \text { locally soluble torsors } C \text { for } E \\
& \text { with degree } p \text { line bundles } L \text { on } C\end{aligned}$ \\
\hline$E(\mathbb{Q}) / p E(\mathbb{Q})$ & $\begin{aligned} \text { pairs }(C, L): & \text { trivial(ized) torsors } C \text { for } E \\
& \text { with degree } p \text { line bundles } L \text { on } C\end{aligned}$ \\
\hline
\end{tabular}

\footnotetext{
${ }^{7}$ A rational degree $p$ divisor on $C$ is equivalent to a formal sum of $p$ points of $C(\overline{\mathbb{Q}})$ which are together defined over $\mathbb{Q}$.
} 
More recently, Bhargava, Kane, Lenstra, Poonen, and Rains BKL ${ }^{+} 13$ have extended these heuristics to model both Selmer groups and Tate-Shafarevich groups simultaneously, by studying the distribution of the exact sequence that is the direct limit over $n$ of (17) with $p$ replaced by $p^{n}$.

There has also been recent progress in studying distributions of 2-Selmer groups for quadratic twist families, including work of Heath-Brown, Swinnerton-Dyer, Kane, Yu, Mazur, Rubin, and Klagsbrun, among many others HB93, HB94, SD08, Kan12,Yu06, Yu05, MR10, KMR11.

Finally, a common theme in arithmetic geometry is to replace a number field by a function field, since geometry often helps in the latter case. Here, if $\mathbb{Q}$ is replaced by the function field $\mathbb{F}_{q}(t)$, de Jong dJ02 gives an upper bound for the average size of 3-Selmer groups of elliptic curves over $\mathbb{F}_{q}(t)$. His methods for parametrizing elements of the Selmer group are similar to those described in 4.1 for Theorem 3.2 (and analogous results for Theorem 5.2).

\section{The AVERAge SIZE of 2-Selmer Groups}

We now explain the main ideas behind Theorem 3.2 and the following stronger statement from BS10a]:

Theorem 4.1 (Bhargava and Shankar 2010). Let $\mathscr{F}$ be any family of elliptic curves $E: y^{2}=x^{3}+A x+B$ defined by finitely many congruence conditions on the integral coefficients $A$ and $B$. Then the average size of $\operatorname{Sel}_{2}(E)$ for elliptic curves $E$ in $\mathscr{F}$, ordered by height, is 3 .

The key observations are that binary quartic forms are closely related to elements of 2-Selmer groups of elliptic curves, and that it is possible to "count" integral binary quartic forms using techniques from the geometry of numbers.

More precisely, we will see in 4.1 that binary quartic forms with rational coefficients, up to standard transformations, with certain local properties correspond exactly to 2-Selmer elements of elliptic curves. The classical invariant theory of binary quartic forms plays a crucial role in this relationship; in particular, it gives the vertical map in diagram (8) below.

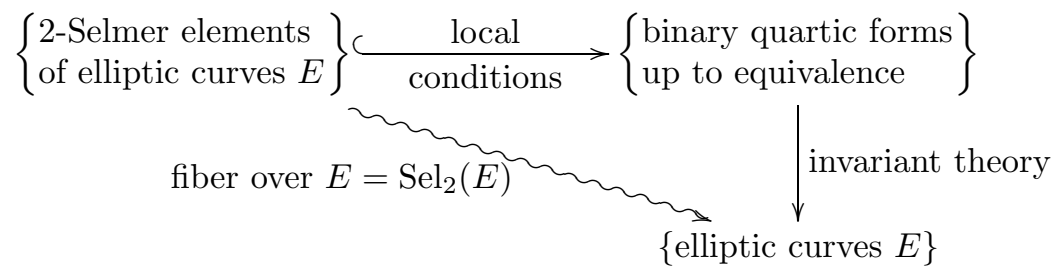

In 84.2 , we explain how suitably enhanced techniques from the geometry of numbers are used to count the number of binary quartic forms with bounded height 8 Incorporating the local conditions by using sieve methods (see 4.3 produces a count of 2-Selmer elements for elliptic curves up to a given height. Because the fiber of the squiggly arrow in diagram (8) above an elliptic curve $E$ is exactly $\operatorname{Sel}_{2}(E)$, dividing this count by the number of elliptic curves up to the same height, and then taking the limit of that quotient as the height tends to infinity, gives the average we seek.

\footnotetext{
${ }^{8}$ The height of a binary quartic form is the same height, up to a constant, as for its associated elliptic curve.
} 
This method is not known to work if the elliptic curves are ordered by discriminant or by conductor, instead of by height; the asymptotic number of elliptic curves with discriminant or conductor less than $X$, as $X$ tends to infinity, is not even known.

4.1. Binary quartic forms and elliptic curves. In the classical work BSD63 of Birch and Swinnerton-Dyer that inspired the BSD conjecture, they study and use the relationship between binary quartic forms and 2-Selmer elements of elliptic curves.

A binary quartic form over $\mathbb{Q}$ is a homogeneous polynomial of degree 4 in two variables with rational coefficients, i.e.,

$$
f\left(x_{1}, x_{2}\right):=a x_{1}^{4}+b x_{1}^{3} x_{2}+c x_{1}^{2} x_{2}^{2}+d x_{1} x_{2}^{3}+e x_{2}^{4}
$$

with $a, b, c, d, e \in \mathbb{Q}$. The set of all binary quartic forms over $\mathbb{Q}$ is a five-dimensional $\mathbb{Q}$-vector space $V$, with coordinates given by the coefficients $a, b, c, d$, and $e$. The group $\mathrm{GL}_{2}(\mathbb{Q})$ acts on the elements of $V$ via

$$
g \cdot f\left(x_{1}, x_{2}\right)=(\operatorname{det} g)^{-2} f\left(\left(x_{1}, x_{2}\right) \cdot g\right)
$$

for all $g \in \mathrm{GL}_{2}(\mathbb{Q})$; since scalar matrices act trivially, this action induces an action of $\mathrm{PGL}_{2}(\mathbb{Q})$. We call two binary quartic forms $f$ and $f^{\prime}$ equivalent if there exists $g \in \mathrm{PGL}_{2}(\mathbb{Q})$ and $\lambda \in \mathrm{GL}_{1}(\mathbb{Q})=\mathbb{Q}^{\times}$such that $f^{\prime}=\lambda^{2}(g \cdot f)$. In other words, the space $V$ is a certain representation of the group $\operatorname{PGL}_{2}(\mathbb{Q}) \times \mathrm{GL}_{1}(\mathbb{Q})$, and two binary quartic forms are equivalent if they are in the same orbit of the group.

Under the action (10) of $\mathrm{GL}_{2}(\mathbb{Q})$, or equivalently, under the induced action of $\mathrm{PGL}_{2}(\mathbb{Q})$, the invariants of a binary quartic form (9) form a polynomial ring generated by two invariants:

$$
\begin{aligned}
& I(f):=12 a e-3 b d+c^{2}, \\
& J(f):=72 a c e+9 b c d-27 a d^{2}-27 b^{2} e-2 c^{3} .
\end{aligned}
$$

The discriminant $\Delta(f):=4 I(f)^{3}-J(f)^{2}$ is nonzero if $f$ has four distinct solutions, up to scaling, over $\overline{\mathbb{Q}}$. The height of $f$ is $\operatorname{ht}(f):=\max \left(\left|I(f)^{3}\right|, J(f)^{2} / 4\right)$.

Genus 1 curves from binary quartic forms. Given a binary quartic form $f\left(x_{1}, x_{2}\right)$ with nonzero discriminant, one may construct a genus 1 curve $C(f)$ explicitly as the smooth compactification of the affine curve

$$
y^{2}=f\left(x_{1}, x_{2}\right) .
$$

This genus 1 curve is the double cover of the projective line ramified at exactly the roots of $f$ (which may not be individually defined over $\mathbb{Q}$ ). It therefore comes equipped with a degree 2 line bundle $L(f)$, namely the pullback of the line bundle $\mathcal{O}(1)$ from $\mathbb{P}^{1}$; equivalently, a rational degree 2 divisor on $C(f)$ is given by the formal sum of the two points in the preimage of any rational point on $\mathbb{P}^{1}$ under this double cover.

If $f^{\prime}$ is an equivalent binary quartic form, then $C\left(f^{\prime}\right)$ and $C(f)$ are isomorphic, and the line bundles for each also correspond to one another under this isomorphism. In fact, binary quartic forms with nonzero discriminant up to equivalence are exactly in one-to-one correspondence with isomorphism classes of genus 1 curves with degree 2 line bundles! 
Moreover, the Jacobian $E(f)$ of $C(f)$ depends only on the two invariants $I(f)$ and $J(f)$; it may be written in short Weierstrass form as

$$
E(f): y^{2}=x^{3}-\frac{I(f)}{3} x-\frac{J(f)}{27} .
$$

Therefore, from our visualization of 2-Selmer elements described in $\$ 3.3$, we see that for a binary quartic form $f$, if $C(f)$ is locally soluble, then the pair $(C(f), L(f))$ (with the action of $E(f)$ on $C(f)$ ) corresponds to an element of $\operatorname{Sel}_{2}(E(f)$ ). More precisely, let $V(\mathbb{Q})^{\text {ls }}$ be the subset of locally soluble binary quartic forms $f\left(x_{1}, x_{2}\right)$ over $\mathbb{Q}$ with $\Delta(f) \neq 0$, i.e., those for which $y^{2}=f\left(x_{1}, x_{2}\right)$ has a $\mathbb{Q}_{\nu}$-solution for all primes $\nu$ (including $\left.\mathbb{Q}_{\infty}=\mathbb{R}\right)$. Note that $V(\mathbb{Q})^{\text {ls }}$ is preserved under the action of $\mathrm{GL}_{2}(\mathbb{Q}) \times \mathbb{Q}^{\times}$.

The equivalence classes of $V(\mathbb{Q})^{1 \text { s }}$ are in correspondence with 2-Selmer elements of elliptic curves; that is, we have the bijection

$$
\mathrm{PGL}_{2}(\mathbb{Q}) \times \mathbb{Q}^{\times} \backslash V(\mathbb{Q})^{\mathrm{ls}} \stackrel{1-1}{\longleftrightarrow}\left\{(E, \zeta): \begin{array}{l}
E \text { elliptic curve } \\
\zeta \in \operatorname{Sel}_{2}(E)
\end{array}\right\} / \cong .
$$

For any specific elliptic curve $E_{A B}: y^{2}=x^{3}+A x+B$, we may specialize to the correspondence

$$
\mathrm{PGL}_{2}(\mathbb{Q}) \backslash V_{A B}(\mathbb{Q})^{1 \mathrm{~s}} \stackrel{1-1}{\longleftrightarrow} \operatorname{Sel}_{2}\left(E_{A B}\right),
$$

where $V_{A B}(\mathbb{Q})^{\text {ls }}$ consists of binary quartic forms $f$ with invariants $I(f)=-3 A$ and $J(f)=-27 B$.

Finding binary quartic forms with specified invariants is the best known way to explicitly compute the 2-Selmer group (and often, the rank) for a given elliptic curve; see, e.g., Cremona's mwrank program Cre12.

Example 4.2. The only rational binary quartic forms, up to the action of $\mathrm{PGL}_{2}(\mathbb{Q})$, with invariants $I=48$ and $J=-432$ are $f_{0}=x_{1}^{4}-6 x_{1}^{2} x_{2}^{2}+4 x_{1} x_{2}^{3}+x_{2}^{4}$ and $f_{1}=x_{1}^{4}+4 x_{1} x_{2}^{3}+4 x_{2}^{4}$. They each have Jacobian isomorphic to the elliptic curve $E$ given by $y^{2}=x^{3}-16 x+16$. Thus,

$$
\operatorname{Sel}_{2}(E) \cong \mathbb{Z} / 2 \mathbb{Z}
$$

with $f_{0}$ representing the identity element. In this case, because $E$ has at least one rational point $(x, y)=(0,4)$ and $E(\mathbb{Q})_{\text {tors }}$ is trivial, the sequence (7) implies that $\operatorname{rk}(E)=1$ and $\amalg(E)[2]=0$.

In order to find the average size of the 2-Selmer group, the goal is therefore to count the number of equivalence classes in $V(\mathbb{Q})^{\mathrm{ls}}$ up to bounded height. The first step is to simply count the number of $\mathrm{PGL}_{2}(\mathbb{Z})$-equivalence classes of binary quartic forms with integral coefficients.

4.2. Counting binary quartic forms using the geometry of numbers. Methods from the geometry of numbers have been previously successful in similar counting questions, such as determining the number of equivalence classes of binary quadratic and binary cubic forms [Mer74, Sie44, Dav51b, Dav51c]. Bhargava and Shankar give an asymptotic count of the number of irreducible integral binary quartic forms, up to equivalence, of bounded height: 
Theorem 4.3 ([BS10a $]$. For $0 \leq i \leq 2$, let $N^{(i)}(X)$ be the number of $\mathrm{PGL}_{2}(\mathbb{Z})$ equivalence classes of irreducible integral binary quartic forms having $4-2 i$ real roots and height less than $X$. Then

$$
\begin{aligned}
& N^{(0)}(X)=\frac{4}{135} \zeta(2) X^{5 / 6}+O\left(X^{3 / 4+\epsilon}\right), \\
& N^{(1)}(X)=\frac{32}{135} \zeta(2) X^{5 / 6}+O\left(X^{3 / 4+\epsilon}\right),
\end{aligned}
$$

and

$$
N^{(2)}(X)=\frac{8}{135} \zeta(2) X^{5 / 6}+O\left(X^{3 / 4+\epsilon}\right) .
$$

One may also impose finitely many congruence conditions on the coefficients $a$, $b, c, d$, and $e$ of the binary quartic forms, e.g., requiring $a$ to be 0 modulo $p$ for a prime $p$. Then the number of equivalence classes of such integral binary quartic forms with height bounded by $X$ is the total number of equivalence classes (the appropriate $N^{(i)}(X)$ from Theorem 4.3) multiplied by the $p$-adic density of each congruence condition imposed, with the same error term of $O\left(X^{3 / 4+\epsilon}\right)$. This $p$-adic density is an easily computable fraction depending on $p$.

Remark 4.4. Although the exact statement of Theorem4.3 is not strictly necessary for the proof of Theorem 4.1, the ideas and results used in the proof of Theorem 4.3 are essentially a subset of those needed for the average Selmer result.

The main idea in proving Theorem 4.3 is to reduce the question to counting lattice points in a nicely shaped domain, in which case the number of lattice points is approximately the volume of the domain. The major complication arises when the domain has cusps, which may be visualized as thin regions going off to infinity. A priori, these cuspidal regions may contain many or few integral points; see Figure 5. A clever "averaging" technique - first introduced by Bhargava in Bha05, Bha10. for asymptotic counts of quartic and quintic rings - helps control exactly which points lie in the cusps.

Let $V^{(i)}$ denote the subset of $V$ corresponding to binary quartic forms with $4-2 i$ roots. For the remainder of this section, we will focus on the case where the binary quartic forms have four real roots (that is, when $i=0$ ); the other two cases are similar.

Reduction theory and fundamental domains. A fundamental domain or set for a group acting on a space is a set of elements in the space containing exactly one representative for each orbit. To count $\mathrm{PGL}_{2}(\mathbb{Z})$-orbits of the space $V(\mathbb{Z})$ of integral binary quartic forms, we may try to count lattice (integral) points in a fundamental
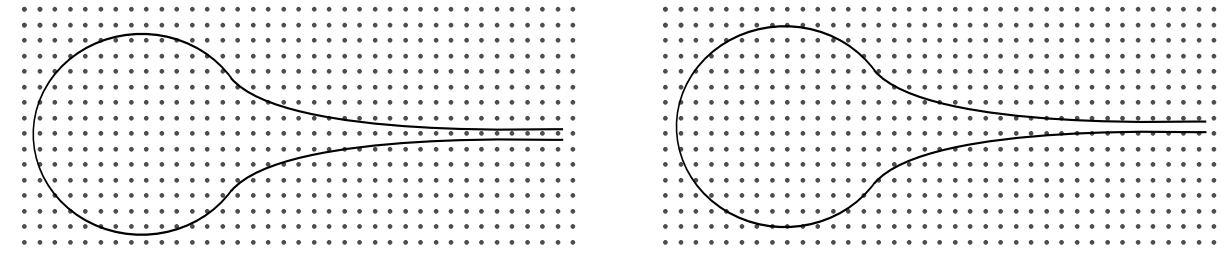

FiguRE 5. A domain with many lattice points in the cusp (left), and a domain with few lattice points in the cusp (right). 
domain for the action of $\mathrm{PGL}_{2}(\mathbb{Z})$ on $V(\mathbb{R})$. It is easier to break up this latter action into two intermediate ones, splitting the problem into two steps:

(i) find a fundamental set for the action of $\mathrm{PGL}_{2}(\mathbb{R}) \times \mathbb{R}^{\times}$on $V(\mathbb{R})$, and

(ii) find a fundamental domain for the action of $\mathrm{PGL}_{2}(\mathbb{Z})$ on $\mathrm{PGL}_{2}(\mathbb{R}) \times \mathbb{R}^{\times}$.

For (i), such a fundamental set is easy to explicitly construct. One checks that a binary quartic form with all real roots and invariants $I$ and $J$ defines a unique $\mathrm{PGL}_{2}(\mathbb{R})$-orbit in $V(\mathbb{R})$ with those invariants. Thus, a fundamental set $L$ consists of real binary quartic forms whose invariants range over all $I$ and $J$, up to scaling (because of the action of $\mathbb{R}^{\times}$). Note that for any $h \in \mathrm{PGL}_{2}(\mathbb{R}) \times \mathbb{R}^{\times}$, the set $h L$ is also a fundamental set. It is crucial that we may choose $L$ such that $h L$ is always a compact set.

Example 4.5. In fact, we may choose representatives for a fundamental set with height 1 . One fundamental set for $V^{(0)}(\mathbb{R})$ is

$$
L=\left\{f_{t}\left(x_{1}, x_{2}\right)=x_{1}^{3} x_{2}-\frac{1}{3} x_{1} x_{2}^{3}-\frac{t}{27} x_{2}^{4}:-2 \leq t \leq 2\right\},
$$

where $I\left(f_{t}\right)=1, J\left(f_{t}\right)=t$, and discriminant $\Delta\left(f_{t}\right)=4-t^{2}>0$.

For (ii), there is a standard decomposition, due to Gauss, of a fundamental domain $\mathcal{F}$ for $\mathrm{PGL}_{2}(\mathbb{Z}) \backslash \mathrm{PGL}_{2}(\mathbb{R}) \times \mathbb{R}^{\times}$. This description also gives explicit coordinates for $\mathcal{F}$.

Combining (i) and (ii) shows that the set $\mathcal{F} h L$, for any $h \in \mathrm{PGL}_{2}(\mathbb{R}) \times \mathbb{R}^{\times}$, contains a representative from each $\mathrm{PGL}_{2}(\mathbb{Z})$-orbit of $V^{(0)}(\mathrm{R})$. In fact, when viewed as a multiset, $\mathcal{F} h L$ overcounts each orbit - by the size of the stabilizer in $\mathrm{PGL}_{2}(\mathbb{R})$ of the binary quartic divided by the size of its stabilizer in $\mathrm{PGL}_{2}(\mathbb{Z})$. For binary quartics in $V^{(0)}(\mathbb{R})$, this quotient is $4 / 1=4$ almost always (in a sense that may be made precise), so it suffices to assume that each orbit is counted four times. In other words, the set $\mathcal{F} h L$ is (almost) a union of four fundamental domains for the action of $\mathrm{PGL}_{2}(\mathbb{Z})$ on $V^{(0)}(\mathbb{R})$.

We are now interested in counting the number of integer points in $\mathcal{F} h L$ of bounded height (and dividing by 4 ).

Averaging and volumes. As alluded to earlier, the number of lattice points in a domain like $\mathcal{F} h L$ is essentially the volume of the domain, by ideas of Minkowski and refinements by Davenport Dav51a, Dav64, but one needs to control the points in the cusp of this domain.

In order to thicken the cusp for better control, we take not just a single domain $\mathcal{F} h L$, but a small ball's worth of such domains by letting the element $h$ vary in a compact set. To obtain the final answer, the number of lattice points in the union of these domains $\mathcal{F} h L$, counted with multiplicity, must be divided by the volume of this compact set.

This larger domain with a thicker cusp may be split into two parts, the main body and the cusp; a clever choice of where to exactly separate the two will give the desired estimates. In particular, let the cusp be the part of the fundamental domain containing the binary quartic forms $f\left(x_{1}, x_{2}\right)$ from (9) for which the absolute value of the coefficient $a$ of $x_{1}^{4}$ is strictly less than 1 . Then any integral binary quartic form in the cusp has $a$ equal to 0 and hence is reducible! 
The volume of the main body then approximates the number of lattice points in it, and one may show that it contains a negligible number of reducible binary quartic forms.

Remark 4.6. Theorem 4.3 only concerns irreducible binary quartic forms, but when we return in 4.3 to counting binary quartic forms corresponding to 2-Selmer elements, we will include the reducible binary quartic forms found in the cusp.

The final step is to compute the volume of this main body, which may be done explicitly. A critical lemma in this computation involves changing from the standard Euclidean measure on the space $V(\mathbb{R})$ to the product of the Haar measure on the group $\mathrm{PGL}_{2}(\mathbb{R})$ and the measures given by the invariants $I$ and $J$. This Jacobian computation mirrors the intuitive idea that $V(\mathbb{R})$ is roughly a product of $\mathrm{PGL}_{2}(\mathbb{R})$ and the quotient $\mathrm{PGL}_{2}(\mathbb{R}) \backslash V(\mathbb{R})$.

4.3. Sieves and uniformity estimates. For Theorems 3.2 and 4.1 , the relevant count is for rational equivalence classes of binary quartic forms corresponding to locally soluble genus 1 curves. Thus, we need to add several steps to the ideas from 4.2

(a) As mentioned in Remark 4.6, the reducible binary quartic forms in the cusp must be incorporated.

(b) Find an integral representative for each $\mathrm{PGL}_{2}(\mathbb{Q}) \times \mathbb{Q}^{\times}$-orbit of $V(\mathbb{Q})^{\text {ls }}$ with integra 9 invariants (and determine exactly how many each rational orbit contains).

(c) Impose the necessary local conditions - via sieve methods - to restrict to the space $V(\mathbb{Q})^{\text {ls }}$ of locally soluble binary quartic forms.

Part (a) is important but straightforward. As mentioned earlier, the cusp region contains binary quartic forms that have a linear factor; these exactly correspond to the identity elements in the 2-Selmer groups! As these and other reducible forms do not appear often in the main body, the main body counts only irreducible binary quartic forms, corresponding to nonidentity elements of the Selmer groups.

The first part of (b) is a standard fact in this case BSD63 CFS10; it is essentially a local computation. That is, given a rational binary quartic form $f$ in $V(\mathbb{Q})^{\text {ls }}$ with integral invariants, then for all primes $p$, there exists an element $g_{p} \in \operatorname{PGL}_{2}\left(\mathbb{Q}_{p}\right)$ such that the binary quartic form $g_{p} \cdot f$ has coefficients in $\mathbb{Z}_{p}$. Then we may use the idea of weak approximation to "glue" together all of these $g_{p}$ 's into an element $g \in \mathrm{PGL}_{2}(\mathbb{Q})$, as $\mathrm{PGL}_{2}$ has class number one; the binary quartic form $g \cdot f$ then has integral coefficients.

In general, however, the orbit of such an $f \in V(\mathbb{Q})^{\text {ls }}$ may contain many $\mathrm{PGL}_{2}(\mathbb{Z})$ orbits, so we need to weight each integral orbit by $1 / n$, where $n$ is the number of integral orbits for that rational orbit. This weighting may in fact be incorporated into the sieve for part (c). Again using the fact that the group $\mathrm{PGL}_{2}$ has class number one, this last step is a local computation; the global weight is a product of local weights, which are related to the size of the stabilizers of the binary quartic forms in $\mathrm{PGL}_{2}\left(\mathbb{Q}_{p}\right)$.

This "geometric sieve", originating in work of Ekedahl Eke91] and extended by Poonen Poo03, Poo04 and Bhargava Bha11, is the final step. Imposing finitely many congruence conditions on the binary quartic forms translates into multiplying

\footnotetext{
${ }^{9}$ For simplicity, we are ignoring some factors of 2 and 3 throughout the discussion of this part.
} 
the original count by the local densities for each condition, as mentioned after Theorem 4.3. However, we now need to impose a condition for every prime $p$, so to obtain an actual limit (as opposed to only a limsup), a certain uniformity estimate is needed 10 In particular, one shows that the binary quartic forms that are "bad" at a prime $p$ are rare as $p$ approaches infinity, so they may be safely ignored.

In the end, the product of all these local factors simplifie 11 to be an invariant of the group $\mathrm{PGL}_{2}$, called the Tamagawa number $\tau\left(\mathrm{PGL}_{2}\right)$. In other words, the limit as $X \rightarrow \infty$ of the weighted number of irreducible integral binary quartic forms in $V(\mathbb{Z})^{\text {ls }}$ with height $<X$, divided by the number of elliptic curves of height $<X$, is $\tau\left(\mathrm{PGL}_{2}\right)=2$. For the average for a family $\mathscr{F}$ of elliptic curves defined by finitely many congruence conditions, the local factors would affect the numerator and denominator equally, so the (limit of the) quotient would not change.

Finally, adding in the cusp contribution (for the identity elements in the 2-Selmer groups) implies the average size of the 2-Selmer group is

$$
2+1=3 .
$$

\section{Generalizations and COROllaries}

We now outline generalizations of Theorem 3.2 and the methods discussed in 44 to other $p$-Selmer groups, other families of elliptic curves, and even families of higher genus curves. In 5.2 , we also explain some corollaries for densities of low rank elliptic curves.

The strategy for proving Theorem 3.2 presented in 4 relies heavily on a description of 2-Selmer elements as equivalence classes of binary quartic forms with certain local properties. The geometry-of-numbers techniques apply to the situation after reducing the question to counting lattice points in a fundamental domain for the action of a group on a vector space.

Generalizing these methods thus depends on relating elements of Selmer groups to the orbits of a vector space $V$ under the action of a group $G$; these orbits may then be counted as before. We modify diagram (8) to reflect the more general goal:

$$
\left\{\begin{array}{l}
p \text {-Selmer elements } \\
\text { for family } \mathscr{F}
\end{array}\right\} \text { conditions }_{\text {fiber }=\text { Sel }_{p}}^{\text {local }}\left\{\begin{array}{l}
G(\mathbb{Q}) \text {-orbits of } V(\mathbb{Q}) \\
\text { counted via geometry of numbers }
\end{array}\right\}
$$

The family $\mathscr{F}$ for Theorem 3.2 consists of elliptic curves in short Weierstrass form. More generally, one may choose $\mathscr{F}$ to be other families of elliptic curves or even higher genus curves, whose Jacobians have analogously defined $p$-Selmer groups.

Finding appropriate groups $G$ and vector spaces $V$ related to the Selmer elements is still a relatively ad hoc process. For elliptic curves, we generally use the geometric

\footnotetext{
${ }^{10}$ In the original paper BS10a, obtaining this uniformity estimate is the most difficult and technical part, but the refined geometric sieve in Bha11 significantly simplifies the computation needed here.

${ }^{11}$ See also Poo12 for an explanation of this fact by computing an adelic volume instead.
} 
description of elements of $p$-Selmer groups - as locally soluble torsors with degree $p$ line bundles-to find such $G$ and $V$.

Remark 5.1. The method summarized in diagram (12) was also previously used by Davenport and Heilbronn DH69] and Bhargava Bha05] to prove two of the only known cases of the Cohen-Lenstra-Martinet heuristics on distributions of ideal class groups of number fields. In those cases, the family $\mathscr{F}$ is replaced by a family of number fields (quadratic fields and cubic fields, respectively, ordered by discriminant), and the $p$-torsion of the ideal class group is the analogue of the $p$-Selmer group.

5.1. Other Selmer groups for elliptic curves. We survey recent results on average sizes of Selmer groups for elliptic curves; the methods behind these theorems all arise from the ideas highlighted in diagram (12).

In BS10b, Bhargava and Shankar extend their methods from BS10a to 3Selmer groups, by using the classical description of 3-Selmer elements as locally soluble curves cut out by ternary cubic forms, up to equivalence.

Theorem 5.2 (Bhargava and Shankar 2010). The average size of the 3-Selmer group for elliptic curves over $\mathbb{Q}$, ordered by height, is 4 .

The average for 3-Selmer groups gives an improved upper bound of $7 / 6$ for the limsup of the average rank of elliptic curves.

In fact, Bhargava and Shankar have work in progress, using similar methods, to show that the average size of the 4- and 5-Selmer groups for elliptic curves, ordered by height, is 7 and 6 , respectively. With some additional work, they are able to use these averages to show that the limsup of the average rank is in fact bounded above by 0.89 .

In joint work with Bhargava $\mathrm{BH} 12$, we find the average sizes of 2- and/or 3Selmer groups for various families of elliptic curves, such as the family

$$
\mathscr{F}_{1}:=\left\{y^{2}+a_{3} y=x^{3}+a_{2} x^{2}+a_{4} x: a_{2}, a_{3}, a_{4} \in \mathbb{Z}, \Delta \neq 0\right\}
$$

of elliptic curves with one marked point, ordered by analogous notions of height. These averages rely on explicit descriptions of Selmer elements for these families as orbits of certain representations $\mathrm{BH} 13$. Upper bounds on average ranks of elliptic curves in these families are also obtained in the same way.

For all the families considered in [BH12, we find that the marked points on the elliptic curves essentially act independently. For example, for the family $\mathscr{F}_{1}$, independence would imply that the single marked point should increase the $p$-rank of the $p$-Selmer group by 1 , and indeed, the 2 - and 3-Selmer groups have average sizes $3 \cdot 2=6$ and $4 \cdot 3=12$, respectively.

5.2. Lots of rank 0 and rank 1 curves. Using the average size of 3 -Selmer groups, one may deduce the existence of many elliptic curves with rank 0 and as a result, many for which BSD is true!

Dokchitser and Dokchitser DD10] prove the $p$-parity conjecture over $\mathbb{Q}$, which states that the root number of an elliptic curve over $\mathbb{Q}$ is determined by the parity of its $p$-Selmer rank. By using congruence conditions to construct a positive-density family of elliptic curves with equidistributed root number, Bhargava and Shankar 
combine the $p$-parity conjecture with Theorem 5.2 to show that a positive density 12 of all elliptic curves, when ordered by height, have rank exactly 0 .

In addition, applying Skinner and Urban's results [SU06, SU10, on the main conjecture of Iwasawa theory for $\mathrm{GL}_{2}$ shows that a positive proportion of elliptic curves have analytic rank exactly 0. Since BSD is known for curves of analytic rank 0 by Kolyvagin's work [Kol88, Bhargava and Shankar conclude that a positive proportion of elliptic curves over $\mathbb{Q}$ satisfy BSD.

Moreover, with the assumption that $\amalg(E)$ for any elliptic curve $E$ is finite (or the weaker assumption that the 3 -torsion subgroup $\amalg(E)[3]$ is always a square), they find a positive density of elliptic curves with rank 1 .

5.3. Higher genus curves. As mentioned in $\$ 2.1$ while curves of genus at least 2 have finitely many rational points, determining the number of rational points is still quite difficult. Given an ordering of curves in a particular family, one may ask similar questions as for elliptic curves; e.g., for any finite number $N$, what is the density of curves with $N$ points? What is the average number of rational points, if finite?

The techniques discussed in s4 surprisingly, give some results towards these questions, at least for hyperelliptic curves. Bhargava and Gross BG12 first find a description of the 2-Selmer elements for hyperelliptic curves with rational Weierstrass points, using rational orbits of a certain representation of odd orthogonal groups (see also the work of Thorne Tho12 for more such parametrizations for higher genus curves using Lie theory). They then compute the average size of the 2-Selmer group:

Theorem 5.3 (Bhargava and Gross 2012). Fix $g \geq 1$. Then the average size of the 2 -Selmer group for Jacobians of genus $g$ hyperelliptic curves over $\mathbb{Q}$ with a rational Weierstrass point, ordered by height, is 3 .

Not only does this give an upper bound of $3 / 2$ for the limsup of the average Mordell-Weil rank of the Jacobians of such curves, but it also may be used-along with the methods of Chabauty and Coleman [Cha41, Col85] - to show that there are many curves with very few points. Poonen and Stoll [PS] have recently improved upon the results from BG12 of this type:

Corollary 5.4 (Poonen and Stoll 2012). Fix $g \geq 3$. Then a positive proportion of genus $g$ hyperelliptic curves over $\mathbb{Q}$ with a rational Weierstrass point have no other rational points, and a majority of such curves have at most seven rational points.

In fact, Poonen and Stoll show that as the genus $g$ tends to infinity, the lower density of these curves for which the given Weierstrass point is the only rational point tends to 1 . Shankar and Wang have obtained similar results for genus $g$ hyperelliptic curves with a marked rational non-Weierstrass point [SW13; they prove an analogue of Theorem 5.3 and apply Poonen and Stoll's techniques to conclude that as $g$ tends to infinity, the lower density of these curves having exactly two rational points (the marked point and its hyperelliptic conjugate) tends to 1.

\footnotetext{
${ }^{12}$ All of these statements on having a positive density or proportion of curves with a given property are, more precisely, about the lower density of such curves being positive.
} 
Finally, Bhargava Bha13 has very recently shown that "most" hyperelliptic curves (not necessarily with a rational point) over $\mathbb{Q}$ have no rational points whatsoever! More precisely, as the genus $g$ tends to infinity, the lower density of hyperelliptic curves over $\mathbb{Q}$ with no rational point tends to 1 .

\section{ACKNOWLEDGMENTS}

This article grew out of notes for the Current Events Bulletin session, organized by David Eisenbud, at the 2013 AMS-MAA Joint Mathematical Meetings. We thank Manjul Bhargava, Bhargav Bhatt, Barry Mazur, Stefan Patrikis, Arul Shankar, and Brian Street for reading and commenting on previous drafts. We thank Nick Katz for suggesting relevant references and the referee for useful comments. All computations were done with SAGE $\left[\mathrm{S}^{+} 12\right.$.

\section{ABOut THE AUTHOR}

Wei Ho received her Ph.D. from Princeton University in 2009. She is currently a Ritt Assistant Professor at Columbia University. Her research interests span topics in number theory, algebraic geometry, and representation theory.

\section{REFERENCES}

[BMSW07] Baur Bektemirov, Barry Mazur, William Stein, and Mark Watkins, Average ranks of elliptic curves: tension between data and conjecture, Bull. Amer. Math. Soc. (N.S.) 44 (2007), no. 2, 233-254, DOI 10.1090/S0273-0979-07-01138-X. MR.2291676 (2009e:11107)

[Bha04a] Manjul Bhargava, Higher composition laws. I. A new view on Gauss composition, and quadratic generalizations, Ann. of Math. (2) 159 (2004), no. 1, 217-250, DOI 10.4007/annals.2004.159.217. MR2051392(2005f:11062a)

[Bha04b] Manjul Bhargava, Higher composition laws. II. On cubic analogues of Gauss composition, Ann. of Math. (2) 159 (2004), no. 2, 865-886, DOI 10.4007/annals.2004.159.865. MR2081442 (2005f:11062b)

[Bha04c] Manjul Bhargava, Higher composition laws. III. The parametrization of quartic rings, Ann. of Math. (2) 159 (2004), no. 3, 1329-1360, DOI 10.4007/annals.2004.159.1329. MR2113024 (2005k:11214)

[Bha05] Manjul Bhargava, The density of discriminants of quartic rings and fields, Ann. of Math. (2) 162 (2005), no. 2, 1031-1063, DOI 10.4007/annals.2005.162.1031. MR2183288(2006m:11163)

[Bha08] Manjul Bhargava, Higher composition laws. IV. The parametrization of quintic rings, Ann. of Math. (2) 167 (2008), no. 1, 53-94, DOI 10.4007/annals.2008.167.53. MR.2373152 (2009c:11057)

[Bha10] Manjul Bhargava, The density of discriminants of quintic rings and fields, Ann. of Math. (2) 172 (2010), no. 3, 1559-1591, DOI 10.4007/annals.2010.172.1559. MR:2745272(2011k:11152)

[Bha11] Manjul Bhargava, The geometric squarefree sieve and unramified nonabelian extensions of quadratic fields, preprint, 2011.

[Bha13] Manjul Bhargava, Most hyperelliptic curves over $\mathbb{Q}$ have no rational points, http:// arxiv.org/abs/1308.0395.

[BG12] Manjul Bhargava and Benedict H. Gross, The average size of the 2-Selmer group of Jacobians of hyperelliptic curves having a rational Weierstrass point, 2012, http:// arxiv.org/abs/1208.1007.

[BH12] Manjul Bhargava and Wei Ho, On the average sizes of Selmer groups in families of elliptic curves, preprint, 2012.

[BH13] Manjul Bhargava and Wei Ho, Coregular spaces and genus one curves, 2013, http:// arxiv.org/abs/1306.4424. 
[BS10a] Manjul Bhargava and Arul Shankar, Binary quartic forms having bounded invariants, and the boundedness of the average rank of elliptic curves, 2010, http://arxiv.org/ abs/1006.1002.

[BS10b] Manjul Bhargava and Arul Shankar, Ternary cubic forms having bounded invariants, and the existence of a positive proportion of elliptic curves having rank 0, 2010, http://arxiv.org/abs/1007.0052.

$\left[\mathrm{BKL}^{+} 13\right]$ Manjul Bhargava, Daniel Kane, Hendrik Lenstra, Bjorn Poonen, and Eric Rains, Modeling the distribution of ranks, Selmer groups, and Shafarevich-Tate groups of elliptic curves, 2013, http://arxiv.org/abs/1304.3971.

[BSD63] B. J. Birch and H. P. F. Swinnerton-Dyer, Notes on elliptic curves. I, J. Reine Angew. Math. 212 (1963), 7-25. MR0146143 (26 \#3669)

[BSD65] B. J. Birch and H. P. F. Swinnerton-Dyer, Notes on elliptic curves. II, J. Reine Angew. Math. 218 (1965), 79-108. MR0179168|(31 \#3419)

[Bom90] Enrico Bombieri, The Mordell conjecture revisited, Ann. Scuola Norm. Sup. Pisa Cl. Sci. (4) 17 (1990), no. 4, 615-640. MR1093712 (92a:11072)

[BCDT01] Christophe Breuil, Brian Conrad, Fred Diamond, and Richard Taylor, On the modularity of elliptic curves over Q: wild 3-adic exercises, J. Amer. Math. Soc. 14 (2001), no. 4, 843-939 (electronic), DOI 10.1090/S0894-0347-01-00370-8. MR 1839918 (2002d:11058)

[Bru92] Armand Brumer, The average rank of elliptic curves. I, Invent. Math. 109 (1992), no. 3, 445-472, DOI 10.1007/BF01232033. MR1176198 (93g:11057)

[BM90] Armand Brumer and Oisín McGuinness, The behavior of the Mordell-Weil group of elliptic curves, Bull. Amer. Math. Soc. (N.S.) 23 (1990), no. 2, 375-382, DOI 10.1090/S0273-0979-1990-15937-3. MR1044170 (91b:11076)

[Cha41] Claude Chabauty, Sur les points rationnels des courbes algébriques de genre supérieur à l'unité, C. R. Acad. Sci. Paris 212 (1941), 882-885 (French). MR0004484 (3,14d)

[CL84] H. Cohen and H. W. Lenstra Jr., Heuristics on class groups of number fields, Number theory, Noordwijkerhout 1983 (Noordwijkerhout, 1983), Lecture Notes in Math., vol. 1068, Springer, Berlin, 1984, pp. 33-62, DOI 10.1007/BFb0099440. MR756082 (85j:11144)

[CM87] H. Cohen and J. Martinet, Class groups of number fields: numerical heuristics, Math. Comp. 48 (1987), no. 177, 123-137, DOI 10.2307/2007878. MR.866103 (88e:11112)

[Col85] Robert F. Coleman, Effective Chabauty, Duke Math. J. 52 (1985), no. 3, 765-770, DOI 10.1215/S0012-7094-85-05240-8. MR808103 (87f:11043)

[CKRS02] J. B. Conrey, J. P. Keating, M. O. Rubinstein, and N. C. Snaith, On the frequency of vanishing of quadratic twists of modular L-functions, Number Theory for the Millennium, I (Urbana, IL, 2000), A K Peters, Natick, MA, 2002, pp. 301-315. MR.1956231 (2003m:11141)

[Cre06] John Cremona, The elliptic curve database for conductors to 130000, Algorithmic number theory, Lecture Notes in Comput. Sci., vol. 4076, Springer, Berlin, 2006, pp. 11-29, DOI 10.1007/11792086_2. MR2282912 (2007k:11087)

[Cre12] John Cremona, mwrank program, 2012, http://homepages.warwick.ac.uk/ masgaj/ mwrank/.

[CR03] J. E. Cremona and D. Rusin, Efficient solution of rational conics, Math. Comp. 72 (2003), no. 243, 1417-1441 (electronic), DOI 10.1090/S0025-5718-02-01480-1. MR1972744 (2004a:11137)

[CFS10] John E. Cremona, Tom A. Fisher, and Michael Stoll, Minimisation and reduction of 2-, 3- and 4-coverings of elliptic curves, Algebra Number Theory 4 (2010), no. 6, 763-820, DOI 10.2140/ant.2010.4.763. MR.2728489|(2012c:11120)

[DW88] Boris Datskovsky and David J. Wright, Density of discriminants of cubic extensions, J. Reine Angew. Math. 386 (1988), 116-138, DOI 10.1515/crll.1988.386.116. MR936994 (90b:11112)

[Dav51a] H. Davenport, On a principle of Lipschitz, J. London Math. Soc. 26 (1951), 179-183. MR0043821 (13,323d)

[Dav51b] H. Davenport, On the class-number of binary cubic forms. I, J. London Math. Soc. 26 (1951), 183-192. MR0043822 (13,323e)

[Dav51c] H. Davenport, On the class-number of binary cubic forms. II, J. London Math. Soc. 26 (1951), 192-198. MR0043823 (13,323f) 
[Dav64] H. Davenport, Corrigendum: "On a principle of Lipschitz", J. London Math. Soc. 39 (1964), 580. MR0166155(29 \#3433)

[DH69] H. Davenport and H. Heilbronn, On the density of discriminants of cubic fields, Bull. London Math. Soc. 1 (1969), 345-348. MR0254010 (40 \#7223)

[Del01] Christophe Delaunay, Heuristics on Tate-Shafarevitch groups of elliptic curves defined over $\mathbb{Q}$, Experiment. Math. 10 (2001), no. 2, 191-196. MR1837670 (2003a:11065)

[Del07] Christophe Delaunay, Heuristics on class groups and on Tate-Shafarevich groups: the magic of the Cohen-Lenstra heuristics, Ranks of Elliptic Curves and Random Matrix Theory, London Math. Soc. Lecture Note Ser., vol. 341, Cambridge Univ. Press, Cambridge, 2007, pp. 323-340.

[DF64] B. N. Delone and D. K. Faddeev, The theory of irrationalities of the third degree, Translations of Mathematical Monographs, Vol. 10, American Mathematical Society, Providence, R.I., 1964. MR.0160744 (28 \#3955)

[DD10] Tim Dokchitser and Vladimir Dokchitser, On the Birch-Swinnerton-Dyer quotients modulo squares, Ann. of Math. (2) 172 (2010), no. 1, 567-596, DOI 10.4007/annals.2010.172.567. MR2680426 (2011h:11069)

[Eke91] Torsten Ekedahl, An infinite version of the Chinese remainder theorem, Comment. Math. Univ. St. Paul. 40 (1991), no. 1, 53-59. MR.1104780 (92h:11027)

[Elk07] Noam Elkies, Three lectures on elliptic surfaces and curves of high rank, 2007, http: // arxiv.org/abs/0709.2908.

[Fal83] G. Faltings, Endlichkeitssätze für abelsche Varietäten über Zahlkörpern, Invent. Math. 73 (1983), no. 3, 349-366, DOI 10.1007/BF01388432 (German). MR718935 (85g:11026a)

[Fal91] Gerd Faltings, Diophantine approximation on abelian varieties, Ann. of Math. (2) 133 (1991), no. 3, 549-576, DOI 10.2307/2944319. MR1109353 (93d:11066)

[Gau01] Carl Friedrich Gauss, Disquisitiones arithmeticae, 1801.

[Gol79] Dorian Goldfeld, Conjectures on elliptic curves over quadratic fields, Number theory, Carbondale 1979 (Proc. Southern Illinois Conf., Southern Illinois Univ., Carbondale, Ill., 1979), Lecture Notes in Math., vol. 751, Springer, Berlin, 1979, pp. 108-118. MR.564926 (81i:12014)

[HB93] D. R. Heath-Brown, The size of Selmer groups for the congruent number problem, Invent. Math. 111 (1993), no. 1, 171-195, DOI 10.1007/BF01231285. MR1193603 (93j:11038)

[HB94] D. R. Heath-Brown, The size of Selmer groups for the congruent number problem. II, Invent. Math. 118 (1994), no. 2, 331-370, DOI 10.1007/BF01231536. With an appendix by P. Monsky. MR1292115 (95h:11064)

[HB04] D. R. Heath-Brown, The average analytic rank of elliptic curves, Duke Math. J. 122 (2004), no. 3, 591-623, DOI 10.1215/S0012-7094-04-12235-3. MR2057019 (2004m:11084)

[dJ02] A. J. de Jong, Counting elliptic surfaces over finite fields, Mosc. Math. J. 2 (2002), no. 2, 281-311. Dedicated to Yuri I. Manin on the occasion of his 65 th birthday. MR:1944508 (2003m:11080)

[KY97] Anthony C. Kable and Akihiko Yukie, Prehomogeneous vector spaces and field extensions. II, Invent. Math. 130 (1997), no. 2, 315-344, DOI 10.1007/s002220050187. MR $1474160(99 \mathrm{c}: 12005)$

[KY02] Anthony C. Kable and Akihiko Yukie, The mean value of the product of class numbers of paired quadratic fields. I, Tohoku Math. J. (2) 54 (2002), no. 4, 513-565. MR.1936267 (2003h:11150)

[Kan12] Daniel M. Kane, On the ranks of the 2-Selmer groups of twists of a given elliptic curve, 2012, http://arxiv.org/abs/1009.1365.

[KS99] Nicholas M. Katz and Peter Sarnak, Random matrices, Frobenius eigenvalues, and monodromy, American Mathematical Society Colloquium Publications, vol. 45, American Mathematical Society, Providence, RI, 1999. MR.1659828 (2000b:11070)

[KS00] J. P. Keating and N. C. Snaith, Random matrix theory and $\zeta(1 / 2+i t)$, Comm. Math. Phys. 214 (2000), no. 1, 57-89, DOI 10.1007/s002200000261. MR1794265 (2002c:11107)

[KMR11] Zev Klagsbrun, Barry Mazur, and Karl Rubin, Selmer ranks of quadratic twists of elliptic curves, 2011, http://arxiv.org/abs/1111.2321. 
[Kol88] V. A. Kolyvagin, Finiteness of $E(\mathbf{Q})$ and $\amalg(E, \mathbf{Q})$ for a subclass of Weil curves, Izv. Akad. Nauk SSSR Ser. Mat. 52 (1988), no. 3, 522-540, 670-671 (Russian); English transl., Math. USSR-Izv. 32 (1989), no. 3, 523-541. MR954295 (89m:11056)

[Maz77] B. Mazur, Modular curves and the Eisenstein ideal, Inst. Hautes Études Sci. Publ. Math. 47 (1977), 33-186 (1978). MR488287 (80c:14015)

[MR10] B. Mazur and K. Rubin, Ranks of twists of elliptic curves and Hilbert's tenth problem, Invent. Math. 181 (2010), no. 3, 541-575, DOI 10.1007/s00222-010-0252-0. MR2660452(2012a:11069)

[Mer74] F. Mertens, Ueber einige asymptotische Gesetze der Zahlentheorie, J. Reine Angew. Math. 77 (1874), 289-338.

[Mor22] Louis J. Mordell, On the rational solutions of the indeterminate equation of the third and fourth degrees, Proc. Cambridge Philos. Soc. 21 (1922), 179-192.

[Poo03] Bjorn Poonen, Squarefree values of multivariable polynomials, Duke Math. J. 118 (2003), no. 2, 353-373, DOI 10.1215/S0012-7094-03-11826-8. MR.1980998 (2004d:11094)

[Poo04] Bjorn Poonen, Bertini theorems over finite fields, Ann. of Math. (2) 160 (2004), no. 3, 1099-1127, DOI 10.4007/annals.2004.160.1099. MR2144974 (2006a:14035)

[Poo12] Bjorn Poonen, Average rank of elliptic curves, Séminaire Bourbaki, 2011-2012, 64ème année no. 1049.

[PR12] Bjorn Poonen and Eric Rains, Random maximal isotropic subspaces and Selmer groups, J. Amer. Math. Soc. 25 (2012), no. 1, 245-269, DOI 10.1090/S0894-03472011-00710-8. MR2833483

[PS] Bjorn Poonen and Michael Stoll, Chabauty's method proves that most odd degree hyperelliptic curves have only one rational point, in preparation.

[SS74] Mikio Sato and Takuro Shintani, On zeta functions associated with prehomogeneous vector spaces, Ann. of Math. (2) 100 (1974), 131-170. MR0344230 (49 \#8969)

[SW13] Arul Shankar and Xiaoheng Wang, Average size of the 2-Selmer group of jacobians of monic even hyperelliptic curves, 2013, http://arxiv.org/abs/1307.3531.

[Sie44] Carl Ludwig Siegel, The average measure of quadratic forms with given determinant and signature, Ann. of Math. (2) 45 (1944), 667-685. MR0012642 (7,51a)

[Sie66] Carl Ludwig Siegel, Über einige Anwendungen diophantischer Approximationen (1929), Gesammelte Abhandlungen. Bände I, II, III, Springer-Verlag, Berlin, 1966, pp. 209-266.

[Sil07] A. Silverberg, The distribution of ranks in families of quadratic twists of elliptic curves, Ranks of elliptic curves and random matrix theory, London Math. Soc. Lecture Note Ser., vol. 341, Cambridge Univ. Press, Cambridge, 2007, pp. 171-176, DOI 10.1017/CBO9780511735158.008. MR2322342 (2008c:11087)

[Sil92] Joseph H. Silverman, The arithmetic of elliptic curves, Graduate Texts in Mathematics, vol. 106, Springer-Verlag, New York, 1992. Corrected reprint of the 1986 original. MR 1329092 (95m:11054)

[SU06] Christopher Skinner and Eric Urban, Vanishing of L-functions and ranks of Selmer groups, International Congress of Mathematicians. Vol. II, Eur. Math. Soc., Zürich, 2006, pp. 473-500. MR2275606(2008a:11063)

[SU10] Christopher Skinner and Eric Urban, The Iwasawa main conjectures for GL(2), preprint. Available at http://www.math.columbia.edu/ urban/eurp/MC.pdf, 2010.

[SW02] William A. Stein and Mark Watkins, A database of elliptic curves - first report, Algorithmic number theory (Sydney, 2002), Lecture Notes in Comput. Sci., vol. 2369, Springer, Berlin, 2002, pp. 267-275, DOI 10.1007/3-540-45455-1_22. MR2041090 (2005h:11113)

[S $\left.\mathrm{S}^{+} 12\right] \quad$ W. A. Stein et al., Sage Mathematics Software (Version 5.3), The Sage Development Team, 2012, http://www.sagemath.org.

[SD08] Peter Swinnerton-Dyer, The effect of twisting on the 2-Selmer group, Math. Proc. Cambridge Philos. Soc. 145 (2008), no. 3, 513-526, DOI 10.1017/S0305004108001588. MR2464773(2010d:11059)

[Tan08] Takashi Taniguchi, A mean value theorem for the square of class number times regulator of quadratic extensions, Ann. Inst. Fourier (Grenoble) 58 (2008), no. 2, 625-670 (English, with English and French summaries). MR2410385 (2009m:11147) 
[TT11] Takashi Taniguchi and Frank Thorne, Secondary terms in counting functions for cubic fields, 2011, http://arxiv.org/abs/1102.2914.

[TW95] Richard Taylor and Andrew Wiles, Ring-theoretic properties of certain Hecke algebras, Ann. of Math. (2) 141 (1995), no. 3, 553-572, DOI 10.2307/2118560. MR1333036 (96d:11072)

[Tho12] Jack A. Thorne, The Arithmetic of Simple Singularities, ProQuest LLC, Ann Arbor, MI, 2012. Thesis (Ph.D.)-Harvard University. MR3054927

[Voj91] Paul Vojta, Siegel's theorem in the compact case, Ann. of Math. (2) 133 (1991), no. 3, 509-548, DOI 10.2307/2944318. MR1109352(93d:11065)

[Wat07] Mark Watkins, Rank distribution in a family of cubic twists, Ranks of Elliptic Curves and Random Matrix Theory, London Math. Soc. Lecture Note Ser., vol. 341, Cambridge Univ. Press, Cambridge, 2007, pp. 237-246, DOI 10.1017/CBO9780511735158.015. MR2322349 (2008k:11072)

[Wat08] Mark Watkins, Some heuristics about elliptic curves, Experiment. Math. 17 (2008), no. 1, 105-125. MR2410120 (2009g:11076)

[Wi195] Andrew Wiles, Modular elliptic curves and Fermat's last theorem, Ann. of Math. (2) 141 (1995), no. 3, 443-551, DOI 10.2307/2118559. MR1333035 (96d:11071)

[WY92] David J. Wright and Akihiko Yukie, Prehomogeneous vector spaces and field extensions, Invent. Math. 110 (1992), no. 2, 283-314, DOI 10.1007/BF01231334. MR $1185585(93 \mathrm{j}: 12004)$

[You06] Matthew P. Young, Low-lying zeros of families of elliptic curves, J. Amer. Math. Soc. 19 (2006), no. 1, 205-250, DOI 10.1090/S0894-0347-05-00503-5. MR2169047 (2006d:11072)

[Yu05] Gang Yu, Average size of 2-Selmer groups of elliptic curves. II, Acta Arith. 117 (2005), no. 1, 1-33, DOI 10.4064/aa117-1-1. MR2110501(2006b:11054)

[Yu06] Gang Yu, Average size of 2-Selmer groups of elliptic curves. I, Trans. Amer. Math. Soc. 358 (2006), no. 4, 1563-1584 (electronic), DOI 10.1090/S0002-9947-05-03806-7. MR2186986 (2006j:11080)

[Yuk93] Akihiko Yukie, Shintani zeta functions, London Mathematical Society Lecture Note Series, vol. 183, Cambridge University Press, Cambridge, 1993. MR.1267735 (95h:11037)

[Yuk97] Akihiko Yukie, Prehomogeneous vector spaces and field extensions. III, J. Number Theory 67 (1997), no. 1, 115-137, DOI 10.1006/jnth.1997.2182. MR.1485429 (99c:12006)

[ZK87] D. Zagier and G. Kramarz, Numerical investigations related to the L-series of certain elliptic curves, J. Indian Math. Soc. (N.S.) 52 (1987), 51-69 (1988). MR989230 (90d:11072)

Department of Mathematics, Columbia University, New York, New York 10027

E-mail address: who@math.columbia.edu 